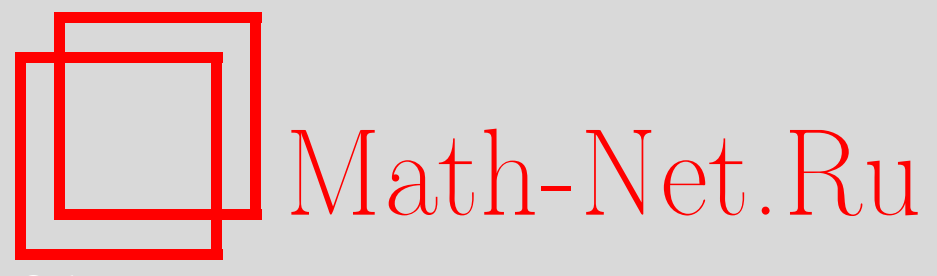

О. В. Шабанин, О сложности дизъюнктивной нормальной формы пороговых функций, Дискрет. матем., 2000, том 12, выпуск 2, 85-92

DOI: https://doi.org/10.4213/dm334

Использование Общероссийского математического портала Math-Net.Ru подразумевает, что вы прочитали и согласны с пользовательским соглашением http://www . mathnet.ru/rus/agreement

Параметры загрузки:

IP : 54.210 .77 .194

26 апреля 2023 г., 13:17:31

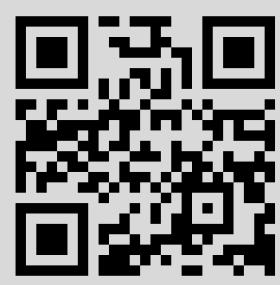




\title{
О сложности дизъюнктивной нормальной формы пороговых функций
}

\author{
(C) 2000 г. О. В. Шабанин
}

\begin{abstract}
В работе рассматривается задача оценки сложности дизъюнктивной нормальной формы (д. н. ф.), понимаемой каќ минимальное число простых импликант, входящих в запись д. н. ф. пороговых функций от $n$ переменных. До этого было известно, что у почти всех пороговых функций сложность д. н. ф. не меньше $n^{2} / \log _{2} n$. В работе доказаны неравенства, связывающие сложность д. н. ф. $L \nu(f)$ пороговой функции $f$ с параметрами Чоу. С их помощью показано, что при достаточно больших $n$ для почти всех пороговых функций
\end{abstract}

$$
\log _{2} L \nu(f)>n-2 \sqrt{2 n \log _{2} n}(1+\delta(n)),
$$

где $\delta(n)-$ любая функция такая, что $\delta(n) \rightarrow 0$ и $n \delta(n) \rightarrow \infty$ при $n \rightarrow \infty$.

Пороговой функцией (см. $[1,2,6])$ называется булева функция

$$
f:\{0,1\}^{n} \rightarrow\{0,1\}
$$

такая, что суцествуют действительные числа $w_{1}, \ldots, w_{n}, T$, удовлетворяющие условию

$$
f\left(x_{1}, x_{2}, \ldots, x_{n}\right)=1 \Longleftrightarrow w_{1} x_{2}+\ldots+w_{n} x_{n} \geqslant T .
$$

При этом будем говорить, что функция $f$ имеет реализацию $\left[w_{1}, \ldots, w_{n} ; T\right]$.

Под сложностью представления произвольной булевой функции в виде дизъюнктивной нормальной формы (д.н.ф.), или просто под сложностью д. н. ф., будем понимать число простых импликант, входящих в запись минимальной д.н.ф. (см. $[7,8])$. Достаточно полный обзор результатов по теории сложности представления булевых функций в различных базисах из функциональных элементов можно найти в работе Р. Г. Нигматуллина [7]. Оценка сложности представления пороговых функций с помощью д.н. ф. представляет теоретический и практический интерес. Хорошо известен факт, что наибольшую сложность д.н.ф., равную $\left(\begin{array}{c}n \\ {[n / 2]}\end{array}\right)$, в классе пороговых функций от $n$ переменных имеет мажоритарная функция (см. [6]). Большая сложность д.н. ф. пороговых функций служит аргументом для использования в ряде случаев пороговых представлений булевых функций вместо представлений в виде д. н.ф. (см. [3]). С другой стороны, исследование сложности д. н. ф. пороговых функций тесно связано с проблемой алгоритмической сложности NP-полных задач. Например, известная задача о ранце: 
максимизировать

$$
\sum_{i=1}^{n} c_{i} x_{i}
$$

при условии

$$
\sum_{i=1}^{n} a_{i} x_{i} \leqslant b,
$$

где $x_{i}$ булевы, $c_{i}, a_{i}, b$ - неотрицательные действительные числа, $i=1, \ldots, n$, может быть решена перебором всех максимальных допустимых решений, верхних нулей соответствующей монотонной функции.

Задача оценки сложности д. н. ф. пороговых функций предложена Ю. А. Зуевым. Ее постановку можно найти, например, в [5]. Наилучшая известная нижняя оценка приведена в работе Ю. А. Зуева и Л. И. Липкина [4], где доказано, что почти у всех пороговых функций сложность д. н. ф. не меньше $n^{2} / \log _{2} n$. В данной работе показано, что при $n \rightarrow \infty$ для почти всех пороговых функций двоичный логарифм сложности д. н. ф. не меньше

$$
n-2 \sqrt{2 \log _{2} n}(1+\delta(n)),
$$

где $\delta(n)$ - любая функция такая, что $\delta(n) \rightarrow 0$ и $n \delta(n) \rightarrow \infty$ при $n \rightarrow \infty$.

Будем считать, что векторы

$$
\left(x_{1}, \ldots, x_{n}\right), \quad\left(y_{1}, \ldots, y_{n}\right), \quad x_{i}, y_{i} \in\{0,1\}, \quad i=1,2, \ldots, n,
$$

связаны соотношением

$$
\left(x_{1}, \ldots, x_{n}\right) \leqslant\left(y_{1}, \ldots, y_{n}\right),
$$

если $x_{i} \leqslant y_{i}$ для всех $i=1,2, \ldots, n$, и соотношением

$$
\left(x_{1}, \ldots, x_{n}\right)<\left(y_{1}, \ldots, y_{n}\right)
$$

если $\left(x_{1}, \ldots, x_{n}\right) \leqslant\left(y_{1}, \ldots, y_{n}\right)$, но $\left(x_{1}, \ldots, x_{n}\right) \neq\left(y_{1}, \ldots, y_{n}\right)$.

Функция $f\left(x_{1}, \ldots, x_{n}\right)$ называется монотонной, если из условия $\left(x_{1}, \ldots, x_{n}\right) \leqslant$ $\left(y_{1}, \ldots, y_{n}\right)$ следует, что $f\left(x_{1}, x_{2}, \ldots, x_{n}\right) \leqslant f\left(y_{1}, y_{2}, \ldots, y_{n}\right)$.

Нижней единицей монотонной функции $f\left(x_{1}, \ldots, x_{n}\right)$ называется вектор $\left(a_{1}, \ldots, a_{n}\right)$ такой, что $f\left(a_{1}, \ldots, a_{n}\right)=1$ и $f\left(b_{1}, \ldots, b_{n}\right)=0$ для любого $\left(b_{1}, \ldots, b_{n}\right)<$ $\left(a_{1}, \ldots, a_{n}\right)$.

Две функции называются однотипными, если одна может быть получена из другой перестановкой координат и заменой некоторых переменных на их отрицания.

Функция $f\left(x_{1}, \ldots, x_{n}\right)$ называется однородной, если существует монотонная функция $g\left(x_{1}, \ldots, x_{n}\right)$, однотипная с функцией $f\left(x_{1}, x_{2}, \ldots, x_{n}\right)$.

Заметим, что если $f$ и $g$ - однотипные функции, то сложности д. н. ф. функций $f$ и $g$ совпадают.

Для монотонной функции $f$ обозначим через $L \nu(f)$ количество нижних единиц функции $f$. Для обобщенно-монотонной функции $g$ пусть $L \nu(g)-$ количество нижних единиц монотонной функции, однотипной с функцией $g$. Корректность такого определения следует из предыдущего замечания.

Известно, что любая пороговая функция является однородной. Учитывая тот факт, что для монотонной функции нижние единицы и только они соответствуют 
простым импликантам в записи д.н.ф. (см. [8]), а также то, что сложности д. н.ф. однотипных функций совпадают, легко показать, что сложность д.н. ф. пороговой функции равна $L \nu(f)$.

Для удобства выкладок перейдем к базису $\{-1,1\}$. Всюда далее рассматриваем функции, отображающие $\{-1,1\}^{n}$ в $\{-1,1\}$. Двоичные функции в базисе $\{-1,1\}$ однозначно соответствуют булевым функциям при замене 0 на -1 . Известно (см. $[1])$, что функция $f$ является пороговой в базисе $\{0,1\}$ тогда и только тогда, когда $f$ - пороговая функция в базисе $\{-1,1\}$. Все введенные выше определения несложно перенести на базис $\{-1,1\}$. При этом останутся верными и сформулированные выше результаты.

Пусть $f\left(x_{1}, x_{2}, \ldots, x_{n}\right)$ - двоичная функция. Введем обозначение

$$
f^{-1}(1)=\left\{\left(a_{1}, a_{2}, \ldots, a_{n}\right) \in\{-1,1\}^{n}: f\left(a_{1}, a_{2}, \ldots, a_{n}\right)=1\right\} .
$$

Целочисленный вектор $\left(\Delta_{0}(f), \Delta_{1}(f), \ldots, \Delta_{n}(f)\right)$ называется характеристическим вектором (вектором Чоу) функции $f$ (см. $[1,2,6])$, если

$$
\begin{aligned}
\Delta_{0} & =2^{n-1}-\left|f^{-1}(1)\right|, \\
\left(\Delta_{1}(f), \ldots, \Delta_{n}(f)\right) & =\sum_{\left(a_{1}, a_{2}, \ldots, a_{n}\right) \in f^{-1}(1)}\left(a_{1}, a_{2}, \ldots, a_{n}\right),
\end{aligned}
$$

где суммирование векторов ведется в поле действительных чисел.

Основное свойство, определяющее исключительное внимание к изучению характеристического вектора в пороговой логике, состоит в следующем (см. $[1,2,6])$ : если $f\left(x_{1}, \ldots, x_{n}\right)$ - пороговая функция, то для любой функции $g\left(x_{1}, \ldots, x_{n}\right), g \neq f$, верно соотношение

$$
\left(\Delta_{0}(g), \Delta_{1}(g), \ldots, \Delta_{n}(g)\right) \neq\left(\Delta_{0}(f), \Delta_{1}(f), \ldots, \Delta_{n}(f)\right) .
$$

Докажем данный факт. Предположим, что выполнено равенство

$$
\left(\Delta_{0}(g), \Delta_{1}(g), \ldots, \Delta_{n}(g)\right)=\left(\Delta_{0}(f), \Delta_{1}(f), \ldots, \Delta_{n}(f)\right)
$$

для некоторой пороговой функции $f$ с реализацией $\left[w_{1}, \ldots, w_{n} ; T\right]$ и произвольной $g$, не равной $f$. Введем обозначения $A=f^{-1}(1)$ и $B=g^{-1}(1)$. Из равенства $\Delta_{0}(g)=$ $\Delta_{0}(f)$ следует, что $|A|=|B|$, а следовательно, $|A \backslash B|=|B \backslash A|$. Остальные равенства дают условие

$$
\sum_{\bar{a} \in A} \bar{a}=\sum_{\bar{a} \in B} \bar{a},
$$

что равносильно равенству

$$
\sum_{\bar{a} \in(A \backslash B)} \bar{a}=\sum_{\bar{a} \in(B \backslash A)} \bar{a} .
$$

Домножим векторы в правой и левой части равенства скалярно на вектор $\left(w_{1}, \ldots, w_{n}\right)$. Тогда по определению пороговой функции левая часть больше либо равна $T|A \backslash B|$, а правая часть меньше, чем $T|A \backslash B|$. Получаем противоречие. Следовательно, исходное утверждение доказано.

Введем обозначения

$$
\begin{aligned}
& f_{0}\left(x_{1}, x_{2}, \ldots, x_{n-1}\right)=f\left(-1, x_{1}, \ldots, x_{n-1}\right) \\
& f_{1}\left(x_{1}, x_{2}, \ldots, x_{n-1}\right)=f\left(1, x_{1}, \ldots, x_{n-1}\right) .
\end{aligned}
$$


Тогда для характеристических векторов

$$
\left(\Delta_{0}\left(f_{0}\right), \Delta_{1}\left(f_{0}\right), \ldots, \Delta_{n}\left(f_{0}\right)\right), \quad\left(\Delta_{0}\left(f_{1}\right), \Delta_{1}\left(f_{1}\right), \ldots, \Delta_{n}\left(f_{1}\right)\right)
$$

функций $f_{0}$ и $f_{1}$ соответственно справедливы равенства

$$
\Delta_{i}(f)=\Delta_{i-1}\left(f_{0}\right)+\Delta_{i-1}\left(f_{1}\right), \quad i=2,3, \ldots, n .
$$

Нетрудно также заметить, что

$$
\left|\Delta_{i}(f)\right| \leqslant 2^{n-1}, \quad i=0,1, \ldots, n .
$$

Пусть $f\left(x_{1}, \ldots, x_{n}\right)$ - пороговая функция с реализацией $\left[w_{1}, \ldots, w_{n} ; T\right]$. Для характеристического вектора

$$
\left(\Delta_{0}(f), \Delta_{1}(f), \ldots, \Delta_{n}(f)\right)
$$

хорошо известны следующие свойства (см. [2]):

$$
\begin{array}{ll}
w_{i} \geqslant 0 \Longrightarrow \Delta_{i}(f) \geqslant 0, & i=1,2, \ldots, n, \\
w_{i} \leqslant 0 \Longrightarrow \Delta_{i}(f) \leqslant 0, & i=1,2, \ldots, n, \\
w_{i} \geqslant w_{j} \Longrightarrow \Delta_{i}(f) \geqslant \Delta_{j}(f), & i, j=1,2, \ldots, n .
\end{array}
$$

Упорядочивая веса пороговой функции, можно дать описание структуры нижних единиц.

Рассмотрим пороговую функцию $f\left(x_{1}, \ldots, x_{n}\right)$ с реализацией

$$
\left[w_{1}, \ldots, w_{n} ; T\right], \quad w_{1} \geqslant w_{2} \geqslant \ldots \geqslant w_{n} \geqslant 0
$$

и условием $f^{-1}(1) \neq\{-1,1\}^{n}$. Тогда вектор $\left(a_{1}, \ldots, a_{k}, 1,-1, \ldots,-1\right)$ является нижней единицей функции $f$ тогда и только тогда, когда

$$
\begin{gathered}
\left(a_{1}, \ldots, a_{k}, 1,-1, \ldots,-1\right) \in f^{-1}(1), \\
\left(a_{1}, \ldots, a_{k},-1,-1, \ldots,-1\right) \notin f^{-1}(1) .
\end{gathered}
$$

Докажем этот факт. Пусть условия (8) выполнены. Предположим, что вектор $\left(a_{1}, \ldots, a_{k}, 1,-1, \ldots,-1\right)$ не является нижней единицей. Тогда существует номер $i, 1 \leqslant i \leqslant n$, такой, что $a_{i}=1$ и

$$
\left(a_{1}, \ldots, a_{i-1},-1, a_{i+1}, \ldots, a_{k}, 1,-1, \ldots,-1\right) \in f^{-1}(1) \text {. }
$$

Так как $w_{i} \geqslant w_{k}$ по условию (1), то

$$
\left(a_{1}, \ldots, a_{i-1}, 1, a_{i+1}, \ldots, a_{k},-1,-1, \ldots,-1\right) \in f^{-1}(1),
$$

получаем противоречие с (8).

В обратную сторону утверждение очевидно по определению нижней единицы.

Следующая лемма связывает сложность д. н. ф. с коэффициентами характеристического вектора пороговой функции. 
Лемма. Пусть $f\left(x_{1}, \ldots, x_{n}\right)$ - пороговая функция с реализацией

$$
\left[w_{1}, \ldots, w_{n} ; T\right], \quad w_{1} \geqslant w_{2} \geqslant \ldots \geqslant w_{n} \geqslant 0 .
$$

Тогда для числа нижсни единич функции $f$ выполняется неравенство

$$
L \nu(f) \geqslant \Delta_{i}(f) / 2^{n-i}, \quad i=1,2, \ldots, n .
$$

Доказательство. Докажем неравенство (10) индукцией по числу переменных.

Для $n=1$ либо $\Delta_{1}(f)=0$, либо $\Delta_{1}(f)=1$, и неравенство (10) очевидно.

Пусть неравенство (10) выполнено для всех пороговых функций с условиями (9) от не более чем $n-1$ переменных. Докажем неравенство для функции от $n$ переменных.

Пусть пороговая функция $f\left(x_{1}, \ldots, x_{n}\right)$ удовлетворяет условиям (9). Тогда

$$
\Delta_{1}(f) \geqslant \Delta_{2}(f) \geqslant \ldots \geqslant \Delta_{n}(f) \geqslant 0 .
$$

Учитывая, что $\left|\Delta_{i}(f)\right| \leqslant 2^{n-1}$, для $i=1$ находим, что

$$
L \nu(f) \geqslant 1 \geqslant \Delta_{1}(f) / 2^{n-1} .
$$

Если $f^{-1}(1)=\{-1,1\}^{n}$, то $\Delta_{1}(f)=\ldots=\Delta_{n}(f)=0$ и оценка (10) верна.

Пусть теперь $i \geqslant 2$ и

$$
f^{-1}(1) \neq\{-1,1\}^{n}
$$

Рассмотрим две функции

$$
\begin{gathered}
f_{-1}\left(x_{1}, x_{2}, \ldots, x_{n-1}\right)=f\left(-1, x_{1}, \ldots, x_{n-1}\right), \\
f_{1}\left(x_{1}, x_{2}, \ldots, x_{n-1}\right)=f\left(1, x_{1}, \ldots, x_{n-1}\right) .
\end{gathered}
$$

Тогда $f_{-1}$ и $f_{1}$ - пороговые функции, как следует из условий (2), (4)-(6), для них

$$
\Delta_{1}\left(f_{\varepsilon}\right) \geqslant \Delta_{2}\left(f_{\varepsilon}\right) \geqslant \ldots \geqslant \Delta_{n}\left(f_{\varepsilon}\right) \geqslant 0, \quad \varepsilon \in\{-1,1\}
$$

и

$$
\Delta_{i}(f)=\Delta_{i-1}\left(f_{0}\right)+\Delta_{i-1}\left(f_{1}\right), \quad i=2,3, \ldots, n
$$

Покажем, что

$$
L \nu(f)=L \nu\left(f_{-1}\right)+L \nu\left(f_{1}\right) .
$$

Для этого достаточно доказать, что вектор $\left(\varepsilon, a_{1}, \ldots, a_{n-1}\right)$ является нижней единицей функции $f$ тогда и только тогда, когда вектор $\left(a_{1}, \ldots, a_{n-1}\right)$ является нижней единицей функции $f_{\varepsilon}, \varepsilon \in\{-1,1\}$.

Пусть $\left(\varepsilon, a_{1}, \ldots, a_{n-1}\right)$ - нижняя единица функции $f$. Определим число $k$ следующим образом: если $a_{n-1}=1$, то $k=n$, если $a_{n-1}=-1$, то $k$ найдем из условия

$$
a_{k}=1, \quad a_{k+1}=a_{k+2}=\ldots=a_{n}=-1 \text {. }
$$

Ввиду условия (11) такое $k$ существует. 
По доказанному выше $\left(\varepsilon, a_{1}, \ldots, a_{k}, 1,-1, \ldots,-1\right)$ - нижняя единица функции $f$ тогда и только тогда, когда

$$
\left(\varepsilon, a_{1}, \ldots, a_{k}, 1,-1, \ldots,-1\right) \in f^{-1}(1),
$$

$\left(\varepsilon, a_{1}, \ldots, a_{k},-1,-1, \ldots,-1\right) \notin f^{-1}(1)$ тогда и только тогда, когда

$$
\left(a_{1}, \ldots, a_{k}, 1,-1, \ldots,-1\right) \in f_{\varepsilon}^{-1}(1)
$$

и

$$
\left(a_{1}, \ldots, a_{k},-1,-1, \ldots,-1\right) \notin f_{\varepsilon}^{-1}(1)
$$

тогда и только тогда, когда $\left(a_{1}, \ldots, a_{k}, 1,-1, \ldots,-1\right)$ - нижняя единица функции $f_{\varepsilon}$. Следовательно, равенство (14) доказано.

Из условий теоремы и свойств $(3),(5)$ и (6) следует, что функции $f_{-1}$ и $f_{1}$, зависящие от $(n-1)$-й переменной, удовлетворяют предположениям индукции. Таким образом, используя равенства (13) и (14), находим, что

$$
\begin{aligned}
L \nu(f) & =L \nu\left(f_{-1}\right)+L \nu\left(f_{1}\right) \geqslant \Delta_{i-1}\left(f_{-1}\right) / 2^{(n-1)-(i-1)}+\Delta_{i-1}\left(f_{1}\right) / 2^{(n-1)-(i-1)} \\
& =\left(\Delta_{i-1}\left(f_{-1}\right)+\Delta_{i-1}\left(f_{1}\right)\right) / 2^{n-i} \geqslant \Delta_{i}(f) / 2^{n-i} .
\end{aligned}
$$

Лемма доказана.

Следствие. Пусть $f\left(x_{1}, \ldots, x_{n}\right)$ - пороговая функция, и пусть

$$
\left|\Delta_{1}^{*}(f)\right| \geqslant\left|\Delta_{2}^{*}(f)\right| \geqslant \ldots \geqslant\left|\Delta_{n}^{*}(f)\right| \geqslant 0
$$

суть числа $\left|\Delta_{1}(f)\right|,\left|\Delta_{2}(f)\right|, \ldots,\left|\Delta_{n}(f)\right|$, расположеннъе в порлдке убивания.

Тогда

$$
L \nu(f) \geqslant\left|\Delta_{i}^{*}(f)\right| / 2^{n-i}, \quad i=1,2, \ldots, n .
$$

Для доказательства основной теоремы понадобится следующий известный результат. Обозначим через $F(n)$ число двоичных пороговых функций от $n$ переменных. В [5] показано, что при $n \rightarrow \infty$

$$
\log _{2} F(n)>n^{2}-n \log _{2} n+O(n) .
$$

Теорема. При $n \rightarrow \infty$ для почти всех пороговьх функций для сложннсти д. н. ф. $L \nu(f)$ въполнено неравенство

$$
\log _{2} L \nu(f)>n-2 \sqrt{2 n \log _{2} n}(1+\delta(n)),
$$

где $\delta(n)$ - любая фунхция такая, что $\delta(n) \rightarrow 0$ u $n \delta(n) \rightarrow \infty$ nрu $n \rightarrow \infty$.

Доказательство. Пусть $f\left(x_{1}, \ldots, x_{n}\right)$ - пороговая функция. Как отмечалось выше, сложность д.н. ф. функции $f$ равна $L \nu(f)$. Пусть функция $\varepsilon(n)$ удовлетворяет условиям $0<\varepsilon(n)<1$ и $\varepsilon(n) \log _{2} n \rightarrow \infty$ при $n \rightarrow \infty$. Положим

$$
p(n)=1-2 \sqrt{(2+\varepsilon(n)) \log _{2} n / n} .
$$

Располагая числа $\left|\Delta_{1}(f)\right|,\left|\Delta_{2}(f)\right|, \ldots,\left|\Delta_{n}(f)\right|$ в порядке убывания, получим последовательность

$$
\left|\Delta_{1}^{*}(f)\right| \geqslant\left|\Delta_{2}^{*}(f)\right| \geqslant \ldots \geqslant\left|\Delta_{n}^{*}(f)\right| \geqslant 0 .
$$


Согласно следствию

$$
L \nu(f) \geqslant\left|\Delta_{i}^{*}(f)\right| / 2^{n-i}, \quad i=1,2, \ldots, n .
$$

Оценим число пороговых функций $H_{1}(n)$ от $n$ переменных, для которых

$$
\left|\Delta_{[n p(n)]}^{*}(f)\right|<2^{n p(n)},
$$

где $[n p(n)]$ - целая часть числа $n p(n)$.

Заметим, что для данной функции $f$ от $n$ переменных количество функций, однотипных с ней (полученных перестановкой переменных и заменой некоторых переменных их отрицаниями), не превосходит $2^{n} n !$. Отсюда следует, что $H_{1}(n) \leqslant 2^{n} n ! H(n)$, где $H(n)$ - количество пороговых функций, удовлетворяющих условиям

$$
\begin{gathered}
\Delta_{1}(f) \geqslant \Delta_{2}(f) \geqslant \ldots \geqslant \Delta_{n}(f) \geqslant 0, \\
\Delta_{[n p(n)]}(f)<2^{n p(n)} .
\end{gathered}
$$

Согласно основному свойству характеристического вектора пороговой функции (1) $H(n)$ не превосходит количества характеристических векторов двоичных функций с ограничениями (16) и (17).

Таким образом, $H(n)$ не превосходит числа всевозможных целочисленных векторов $\left(\Delta_{0}, \Delta_{1}, \Delta_{2}, \ldots, \Delta_{n}\right)$ с ограничениями

$$
\begin{aligned}
& 0 \leqslant \Delta_{i} \leqslant 2^{2 n(p)}, \quad i=[n(p(n)],[n p(n)]+1, \ldots, n, \\
& 0 \leqslant \Delta_{i} \leqslant 2^{n-1}, \quad i=1,2, \ldots,[n(p(n)]-1, \\
& -2^{n-1} \leqslant \Delta_{0} \leqslant 2^{n-1} \text {. }
\end{aligned}
$$

Следовательно,

$$
H(n) \leqslant\left(2^{n}+1\right)\left(2^{n-1}+1\right)^{[n p(n)]} 2^{n p(n)(n-[n p(n)])} .
$$

Отсюда,

$$
\log _{2} H(n) \leqslant n^{2} p(n)+n p(n)(n-n p(n)+1)+\log _{2} n .
$$

При $n \rightarrow \infty$ разность

$$
\begin{aligned}
\log _{2} H_{1}(n)-\log _{2} F(n)< & \left(n^{2} p(n)+n p(n)(n-n p(n)+1)+\log _{2} n+n+n \log _{2} n\right) \\
& -\left(n^{2}-n \log _{2} n+o\left(n \log _{2} n\right)\right) \\
= & \left.-n^{2}(1-p(n))^{2}+n p(n)+2 n \log _{2} n+O(n)\right) \\
= & -\varepsilon(n) n \log _{2} n+O(n)
\end{aligned}
$$

стремится к $-\infty$ согласно выбору $\varepsilon(n)$.

Отсюда следует, что $H_{1}(n) / F(n) \rightarrow 0$ при $n \rightarrow \infty$.

Таким образом, показано, что для почти всех пороговых функций выполняется неравенство

$$
\left|\Delta_{[n p(n)]}^{*}(f)\right| \geqslant 2^{n p(n)} .
$$

Согласно следствию

$$
L \nu(f) \geqslant \Delta_{[n p(n)]}^{*}(f) / 2^{n-[n p(n)]} .
$$


Тогда из (18) и (19) следует, что для почти всех пороговых функций

$$
L \nu(f) \geqslant 2^{n p(n)} / 2^{n-n p(n)} \geqslant 2^{n(2 p(n)-1)} .
$$

Следовательно,

$$
\log _{2} L \nu(f) \geqslant n\left(1-2 \sqrt{(2+\varepsilon(n)) \frac{\log _{2} n}{n}}\right) .
$$

Теорема доказана.

\section{Список литературы}

1. Бутаков Е. А., Методы синтеза релейных устройств из пороговых элементов. Энергия, Москва, 1970.

2. Дертоузос М., Пороговая логика. Мир, Москва, 1967.

3. Зуев Ю. А., Пороговые функции и пороговые представления булевых функций. Математические вопроси кибернетихи (1994) 5, 5-61.

4. Зуев Ю. А., Липкин Л. И., Регулярные булевы функции с заданной сложностью дезъюнктивных нормальных форм. Методы дискретного анализа в изучении булевых функций и графов. ИМ СО АН СССР, Новосибирск, 1989, 48, 17-22.

5. Зуев Ю. А., Асимптотика логарифма числа пороговых функций алгебры логики. Докл. AH CCCP (1989) 306, №3, 528-530.

6. Muroga S., Threshold Logic and its Applications. Wiley, New York, 1971.

7. Нигматуллин Р. Г., Сложностъ булевљх функций. Наука, Москва, 1990.

8. Яблонский С. В., Введение в дискретную математику. Наука, Москва, 1979.

9. Яблонский С. В., Дискретная математиха и математическая кибернетиха. Наука, Москва, 1974.

Статья поступила 17.05.1999. 